\title{
Treatment with Soluble VEGF Receptor Reduces Disease Severity in Murine Collagen-Induced Arthritis
}

\author{
Jadwiga Miotla, Rose Maciewicz, Jane Kendrew, Marc Feldmann, and \\ Ewa Paleolog
}

Kennedy Institute of Rheumatology (JM, MF, EP), London, and AstraZeneca Pharmaceuticals (RM, JK), Alderley Park, Macclesfield, Cheshire, United Kingdom

\begin{abstract}
SUMMARY: Maintenance of the invasive pannus in rheumatoid arthritis is an integral part of disease progression. The synovial vasculature plays an important role in the delivery of nutrients, oxygen, and inflammatory cells to the synovium. Vascular endothelial growth factor (VEGF), an endothelial mitogen expressed by cells within the synovial membrane, is thought to contribute to the formation of synovial blood vessels. Our objective in this study was to measure the kinetics of VEGF production in a murine model of collagen-induced arthritis and to determine whether VEGF blockade reduces disease progression. Synovial cells isolated from the knee joints of naive or sham-immunized mice, or from mice immunized with collagen but without arthritis, released little or no detectable VEGF. Onset of arthritis was associated with expression of VEGF mRNA and protein. The levels of VEGF secreted by synovial cells isolated from the joints of mice with severe arthritis were significantly higher than from mice with mild disease. To block VEGF activity, animals were treated after arthritis onset with a soluble form of the Flt-1 VEGF receptor (sFlt), which was polyethylene glycol (PEG)-linked to increase its in vivo half-life. Treatment of arthritic mice with sFlt-PEG significantly reduced both clinical score and paw swelling, compared with untreated or control-treated (heat-denatured sFlt-PEG) animals. There was also significantly less joint inflammation and reduced bone and cartilage destruction in sFlt-PEG-treated animals, as assessed by histology. Our data demonstrate that, in collagen-induced arthritis, expression of the potent angiogenic cytokine VEGF correlates with disease severity. Furthermore, specific blockade of VEGF activity results in attenuation of arthritis in both macroscopic and microscopic parameters. These observations indicate that blood vessel formation is integral to the development of arthritis and that blockade of VEGF activity might be of therapeutic benefit in rheumatoid arthritis. (Lab Invest 2000, 80:1195-1205).
\end{abstract}

$R$ heumatoid arthritis (RA) is a disabling chronic inflammatory disease that causes progressive joint destruction, deformity, disability, and premature death. The main features of RA are an inflamed synovial lining of the joint spaces, characterized by marked leukocyte infiltration and synovial cell proliferation. An inflammatory pannus eventually invades and destroys the underlying cartilage and bone, leading to severe pain and impairment of mobility (Feldmann et al, 1996). The majority of the manifestations of RAinflammation, joint swelling, bone and cartilage degeneration, and pain-can be attributed to the pannus-derived inflammatory cytokines, chemokines, and destructive enzymes such as matrix metalloproteases. It is therefore not unreasonable to suggest that suppression of pannus growth would be a beneficial element of antiarthritic therapy.

One potential method of attenuating development of the pannus is to interfere with its blood supply, which plays a key role in maintaining the pannus through the supply of nutrients and oxygen necessary for synovial cell proliferation and also in the delivery of inflammatory cells and molecules to the lesion. Furthermore, as a result of the synovial hyperplasia and

Received February 15, 2000.

Address reprint requests to: $\operatorname{Dr}$ E. M. Paleolog, Kennedy Institute of Rheumatology, 1, Aspenlea Road, Hammersmith, London W6 8LH, United Kingdom. Fax: 440208383 4499; E-mail: e.paleolog@ic.ac.uk increased metabolic demand that occur during RA, existing blood vessels may become distant from the growing mass of the arthritic tissue that invades the intra-articular space, leading to regions of perfusion insufficiency. This results in compensatory new blood vessel formation (angiogenesis), thus further promoting and sustaining the invasive and inflammatory processes (Koch, 1998; Paleolog and Fava, 1998; Paleolog and Miotla, 1998). In addition, the vascular endothelial lining of synovial blood vessels appears to exhibit an activated phenotype, with increased expression of adhesion molecules, such as vascular cell adhesion molecule-1, intercellular adhesion molecule-1, E-selectin, and vascular adhesion protein-1 (Salmi et al, 1997), which would preferentially direct the extravasation of pathologically important effector cells into inflamed synovium. Targeting the vasculature in RA would thus potentially diminish delivery of cells, nutrients, and inflammatory mediators, as well as reduce the extravasation and survival of inflammatory cells into the synovial tissue.

The potent angiogenic cytokine vascular endothelial growth factor (VEGF), which is highly up-regulated in RA synovium, is thought to play a role in angiogenesis-dependent diseases such as RA (Brown et al, 1997; Neufeld et al, 1999). In addition to stimulating endothelial cell proliferation and chemotaxis, VEGF increases vascular permeability and could potentially play a role not only in synovial angiogenesis 
but also in joint swelling (Paleolog, 1996). VEGF has been detected in synovial fluids from RA patients and is expressed by RA synovial macrophages and lining cells, as well as by endothelial cells lining small blood vessels within the pannus (Fava et al, 1994; Koch et al, 1994; Nagashima et al, 1995). Moreover both VEGF receptors (Flt-1 and KDR) are expressed on microvascular endothelial cells within the RA synovium (Fava et al, 1994). Recent studies from our laboratory have demonstrated that serum concentrations of VEGF are markedly greater in patients with RA when compared with nonarthritic control subjects and correlate with markers of disease activity. In addition, treatment with anti-tumor necrosis factor- $\alpha$ (anti-TNF $\alpha$ ) monoclonal antibody, which ameliorates disease, significantly reduced VEGF levels in the serum, in parallel with decreased synovial vascularity (Paleolog et al, 1998; Taylor et al, 1998). We have also observed that cells isolated from RA synovium respond to both hypoxia and inflammatory cytokines [TNF $\alpha$ and interleukin-1 (IL-1)] to up-regulate VEGF release, suggesting that formation of new blood vessels in the RA pannus may be driven by both hypoxia- and inflammation-induced expression of VEGF (Paleolog et al, 1998).

In view of our observation that the beneficial effect of anti-TNF $\alpha$ monoclonal antibody in patients with RA is associated with a reduction in serum VEGF levels in RA patients, it is conceivable that specific and selective blockade of VEGF activity in vivo could have an inhibitory effect on arthritis progression. The aim of the present study was to characterize the time course of VEGF production during the development of disease, using the mouse model of collagen-induced arthritis (CIA). The CIA model is an important model of RA because it has a similar profile of cytokine production during disease progression and it exhibits comparable synovial inflammation and cartilage/bone destruction in histological assessment. Use of the model has led to the successful development of anti-TNF $\alpha$ antibody (infliximab) for treatment of RA in the clinical setting. For these reasons, we have characterized the production of VEGF from synovial cells isolated from knee joints at various stages of $\mathrm{CIA}$, and compared this production with the clinical score and paw swelling, which are measures of the severity of CIA disease. In addition, we have investigated the role of VEGF in the development of arthritis by antagonism of VEGF effects in vivo, using a polyethylene glycol (PEG)-linked soluble form of the Flt-1 receptor. We demonstrate that expression of VEGF correlates with disease parameters such as paw swelling and that blockade of VEGF activity markedly attenuates arthritis. These observations suggest that anti-VEGF treatment could be considered as an adjunct for the management of patients with RA.

\section{Results}

\section{Time Course of VEGF Expression from Synovial Cells}

The synovial membrane was dissected from the knee joints of mice with or without arthritis and the cells were cultured in order to measure VEGF production. In control animals without arthritis (either naive or shamimmunized, age-matched mice), the synovial membrane dissected from knee joints was devoid of significant cellular infiltrate. The isolated cells did not produce detectable VEGF after 24 hours in culture (Table 1). Similarly, after immunization with collagen, but in the absence of clinical manifestation of arthritis (Days 14 and 21 post-immunization), isolated synovial cells produced very low levels of VEGF.

Immunization with collagen led to the development of arthritis as manifested by paw swelling, with a median day onset of 25 days post-immunization. The severity of arthritis increased progressively over 10 days as reflected by both clinical score and paw swelling. On the day of disease onset, ie, Day 1 of arthritis, the synovial membrane appeared infiltrated with inflammatory cells (a mixed population of polymorphonuclear cells, macrophages, and lymphocytes), and the total number of cells isolated from the synovium was increased compared with that obtained from the knee joints of healthy mice (not shown). Although there was evidence of arthritis, VEGF production by synovial cells, cultured at a fixed cell density of $4 \times 10^{6} / \mathrm{ml}$ in $30 \mathrm{~mm}^{2}$ wells, remained at levels similar to those measured prior to disease onset (Table 1). However, by Day 5 of disease, there was a marked increase in VEGF protein, as detected by ELISA, with VEGF release equivalent to $263 \pm 114$ $\mathrm{pg} / \mathrm{ml}$ (Table 1). The amount of VEGF produced in-

Table 1. Release of VEGF from Dissociated Synovial Membrane Cells from Arthritic, But Not Healthy, Mice ${ }^{a}$

\begin{tabular}{llcc}
\hline \multicolumn{1}{c}{ Treatment } & \multicolumn{1}{c}{ Time } & $\begin{array}{c}\text { Number of } \\
\text { mice }\end{array}$ & VEGF (pg/ml) \\
\hline Naive & & 10 & $6 \pm 6$ \\
Sham-immunized & & 7 & $6 \pm 4$ \\
Collagen-immunized & Day 14 post-immunization & 5 & $22 \pm 15$ \\
& Day 21 post-immunization & 5 & $27 \pm 12$ \\
& Day 1 of arthritis & 11 & $263 \pm 15$ \\
& Day 5 of arthritis & 25 & $445 \pm 134^{\star *}$ \\
\hline
\end{tabular}

\footnotetext{
VEGF, vascular endothelial growth factor.

${ }^{a}$ Synovial membrane cells were cultured at a density of $4 \times 10^{6}$ cells $/ 30 \mathrm{~mm}^{2}$ wells in flat-bottomed plates for 24 hours. VEGF levels in culture supernatants were determined by ELISA. Values are mean $\mathrm{pg} / \mathrm{ml} \pm$ SEM. Statistical analyses were performed using an unpaired $t$-test with Welch's correction: * $p<0.05 ;{ }^{*} \mathrm{p}<0.01$ versus VEGF release from sham-immunized mice.
} 
creased as arthritis progressed, with maximal release observed on Day 10 of disease ( $p<0.01$ vs VEGF release from healthy mice and on Day 1 of arthritis by unpaired $t$ test with Welch's correction) (Table 1). If the disease was followed beyond 10 days, there was little further alteration in the clinical score or paw swelling. Synovial cells isolated from the knee joints of mice between Days 20 and 25 after arthritis onset continued to secrete significant amounts of VEGF, equivalent to $400 \pm 120 \mathrm{pg} / \mathrm{ml}(n=8)$.

The expression of VEGF by synovial cells isolated from arthritic, but not healthy mice, was confirmed by PCR for VEGF mRNA (Fig. 1). Comparison of mRNA samples obtained from the knees of mice killed on Day 5 after disease onset revealed that synovial cells from diseased animals contained expressed mRNA encoding for VEGF-164 and VEGF-120 isoforms (570 bp and 438 bp PCR products respectively) (Claffey et al, 1992; Shima et al, 1996). Similar bands were observed in extracts from the mouse macrophage cell line RAW264.7, which had been exposed to hypoxia for 24 hours as previously described (Paleolog et al, 1998; Vasir et al, 1998).

\section{Synovial Cell VEGF Correlates with Paw Swelling and Clinical Score}

To assess whether the extent of VEGF produced by cells from knee joints of arthritic mice was dependent on the severity of disease, the amount of VEGF secreted by cultures from individual animals was compared with paw thickness and clinical score on the day of death (Fig. 2A and Fig. 3A, respectively). The Spearman coefficient for a nonparametric correlation was calculated, and the values were found to be $r=$ $0.29(p<0.05)$ and $r=0.39(p<0.01)$ for paw thickness and clinical score, respectively.

To further analyze the data, we compared VEGF release by synovial cells isolated from mice with mild disease and severe disease. The median paw thickness value was $2.4 \mathrm{~mm}$, so mild disease was defined
(A)

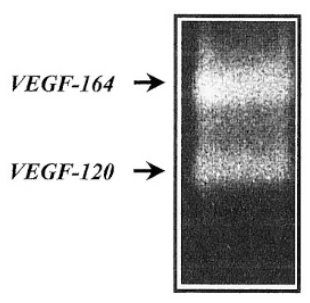

(B)

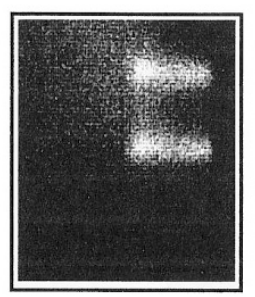

Naive Arthritic
(C)

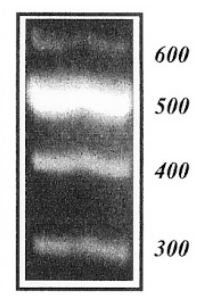

(B)

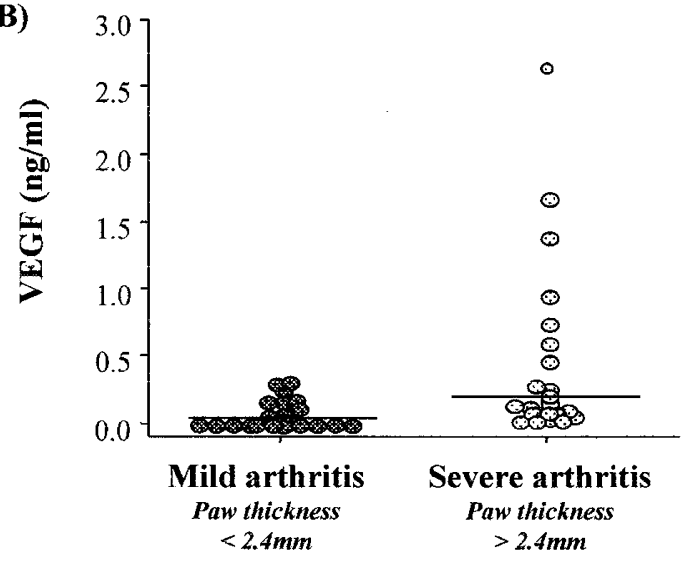

Figure 2.

VEGF release by synovial cells from arthritic mice correlates with paw thickness. Synovial membrane cells were cultured at a density of $4 \times 10^{6}$ cells/30 mm² wells for 24 hours. VEGF levels in culture supernatants were determined by ELISA. A, Correlation of VEGF release with paw thickness on the day of sacrifice. B, Comparison of VEGF levels in culture supernatants from mice with mild disease (defined as paw thickness equal to or less than the median value of $2.4 \mathrm{~mm} ; n=28$ ) and severe disease (paw thickness greater than the median value of $2.4 \mathrm{~mm} ; n=24$ ). Bars indicate median values for VEGF release.

as paw thickness equal to or less than $2.4 \mathrm{~mm}(n=$ 28 ), whereas severe disease was defined as greater than $2.4 \mathrm{~mm}(n=24)$ (Figure 2B). The amounts of VEGF produced by cells from the two different groups were found to be significantly different: $20 \mathrm{pg} / \mathrm{ml}$ VEGF for mice with mild disease and $190 \mathrm{pg} / \mathrm{ml}$ for mice with severe arthritis ( $p=0.01$ vs VEGF release by synovial cells from mice with paw thickness $<2.4 \mathrm{~mm}$ by Mann-Whitney $U$ test). Similarly, we used clinical score data to subdivide groups of animals into mice with mild disease (clinical scores equal to or less than the median value of $3 ; n=29$ ) and mice with severe disease (mice with clinical scores greater than the median value of $3 ; n=23$ ). VEGF release by synovial cells isolated from mice with clinical scores equal to or less than the median value was $30 \mathrm{pg} / \mathrm{ml}$, compared with $260 \mathrm{pg} / \mathrm{ml}$ for mice with clinical scores greater than the median value $(p<0.01$ vs VEGF release by
Figure 1.

Vascular endothelial growth factor (VEGF) mRNA is expressed by synovial cells from arthritic, but not healthy mice. A, Polymerase chain reaction amplification of VEGF mRNA isolated from murine macrophage cell line RAW 264.7, exposed to hypoxia for 24 hours as previously described (Paleolog et al, 1998) B, Synovial membrane cells prepared from the knees of either healthy $(n=5)$ or arthritic mice ( $n=5$; Day 5 after onset of disease symptoms). C, Standard DNA ladder of 100-660 bp. All samples were probed and standardized for mouse GAPDH mRNA (not shown). 
(A)

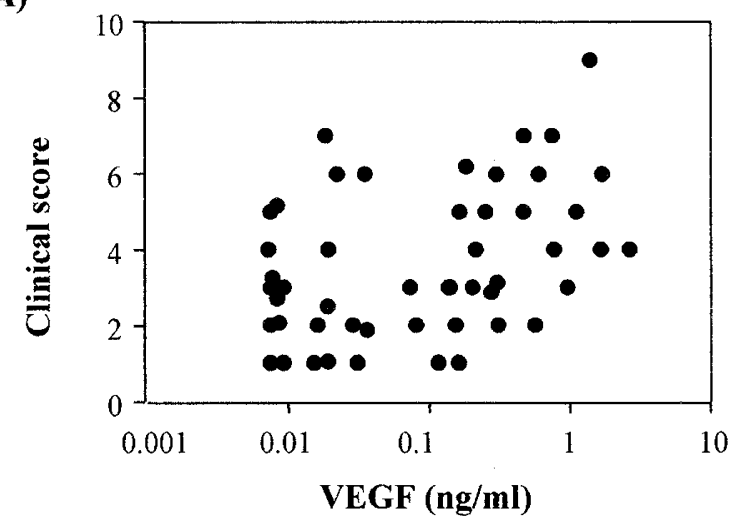

(B)

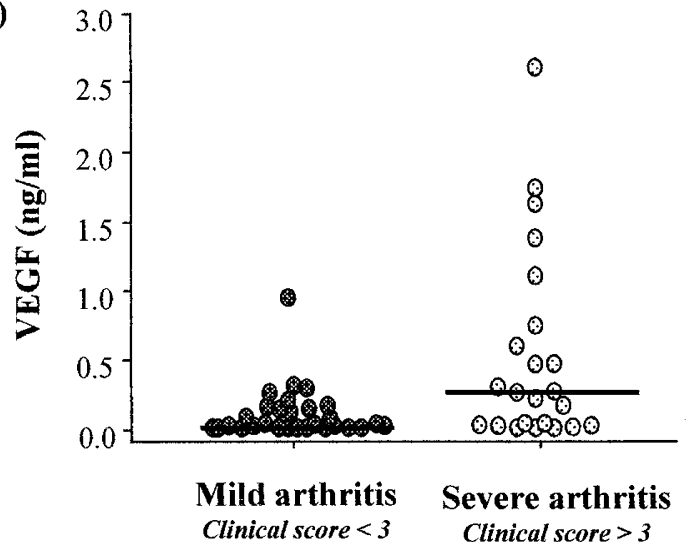

Figure 3.

VEGF release by synovial cells from arthritic mice correlates with clinical score. Synovial membrane cells were cultured at a density of $4 \times 10^{6}$ cells $/ 30 \mathrm{~mm}^{2}$ wells for 24 hours. VEGF levels in culture supernatants were determined by ELISA. A, Correlation of VEGF release with clinical score on the day of sacrifice. $B$, Comparison of VEGF levels in culture supernatants from mice with mild disease (clinical scores equal to or less than the median value of $3 ; n=29$ ) and severe disease (mice with clinical scores greater than the median value of $3 ; n=23$ ). Bars indicate median values for VEGF release.

synovial cells from mice with clinical score $<3$ by Mann-Whitney $U$ test) (Fig. 3B).

\section{Soluble VEGF Receptor sFlt-PEG Reduces Disease Severity in CIA}

The effect of VEGF blockade in CIA was assessed using PEG-linked soluble Flt-1 (sFlt-PEG) after disease onset. Animals immunized with type II collagen according to the usual protocol were treated with sFIt-PEG from the first day of arthritis onset and compared with treatment with heat-denatured sFItPEG or with PBS as the vehicle control. A control group of animals was untreated throughout 10 days of arthritis. We observed that treatment with sFIt-PEG at a dose of $100 \mu \mathrm{g}$ every other day until Day 10 had no significant effect on CIA progression or severity of disease (data not shown). The clinical score and extent of paw swelling were not affected by treatment and did not significantly differ from control arthritic mice or those treated with denatured compound.

In contrast, daily administration of sFIt-PEG at the higher dose of $300 \mu \mathrm{g}$ per injection resulted in a marked attenuation of CIA. Treatment with sFIt-PEG from the day of arthritis onset until Day 5 suppressed the increase in clinical score compared with control groups (Fig. 4A). Administration of heat-denatured sFIt-PEG had no significant effect on clinical score compared with either untreated or PBS-treated arthritic animals. Although treatment with sFIt-PEG was ceased on Day 5 , the inhibitory effect was observed throughout the 10 days of the experiment $(p<0.001$ vs untreated and denatured sFIt-PEG-treated mice by ANOVA). For example, when the clinical scores on Day 10 of arthritis were compared, the scores were $3.9 \pm$ 0.4 (mean $\pm \mathrm{SEM}, n=16$ ) and $3.7 \pm 0.6(n=15)$ for untreated and denatured sFlt-PEG-treated mice, re-

(A)

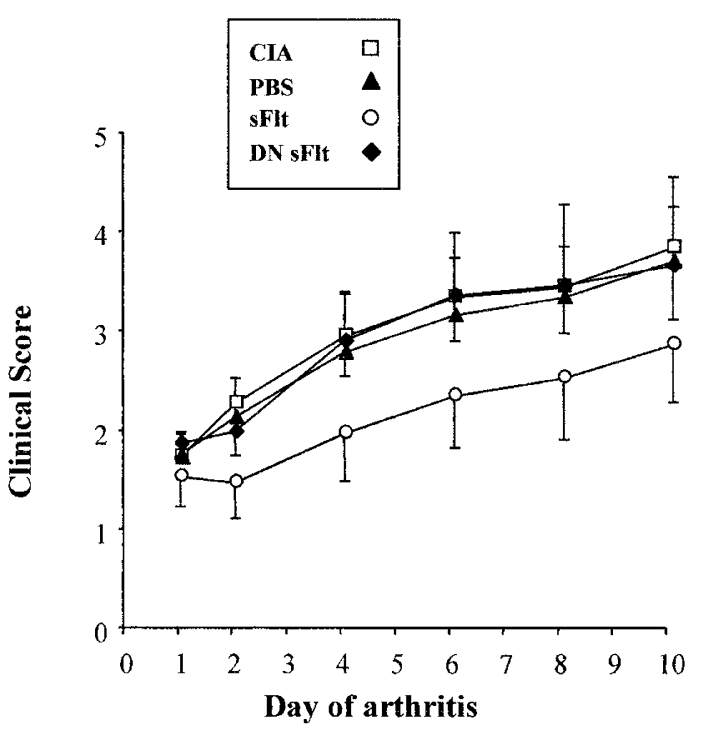

(B)

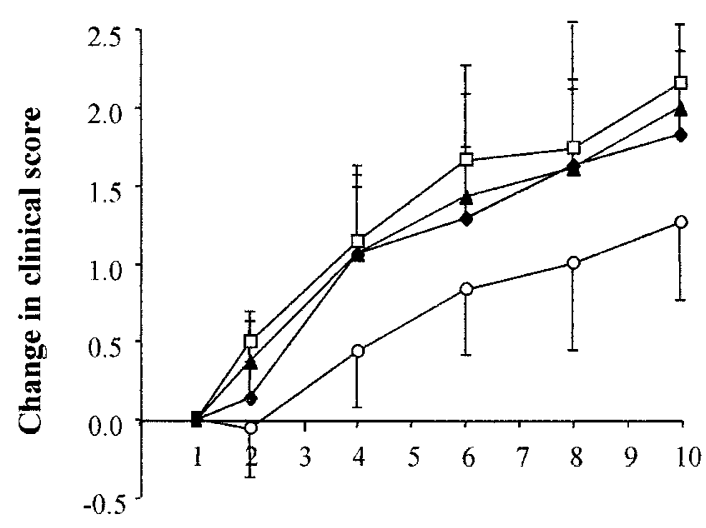

Day of arthritis

Figure 4.

Soluble VEGF receptor reduces the clinical score in arthritic mice. Arthritis was induced in mice by immunization with bovine collagen. From the day of arthritis onset, mice were either untreated $(\square, n=16)$ or received the following treatments: sFlt-PEG $(\bigcirc, n=15)$ or denatured (DN) sFlt-PEG ( $\bullet$, $n=15)$, both at a dose of $300 \mu \mathrm{g}$, or vehicle $(\boldsymbol{\Delta}$, PBS, $n=8)$, ip daily until Day 5 of arthritis. A, The clinical score was monitored daily until Day 10 or, B, expressed as change from Day 1 . Results are mean \pm SEM. 
spectively. In comparison, the clinical score in sFltPEG-treated mice was $2.9 \pm 0.6(n=15)$. Since the treatments were given randomly, the mean clinical scores on the day of arthritis onset were found to be different among the various groups. In order to normalize the data, the change in clinical scores relative to Day 1 was therefore calculated for each group. After administration of sFlt-PEG, the increase in the clinical score was reduced compared with the change seen in all control groups (Fig. 4B).

The effect of treatment on paw swelling was also examined (Fig. 5). Treatment with sFlt-PEG markedly reduced the paw swelling when compared with the group of mice who received the heat-denatured form of sFlt-PEG ( $p<0.001$ vs denatured sFlt-PEG-treated mice by ANOVA) (Fig. 5A). However, it should be noted that the mean paw swelling in the sFlt-PEG-treated group on the day of arthritis onset was significantly greater $(p<0.01)$ than the paw swelling in untreated or PBS-treated animals. This highlights the necessity for normalizing the data relative to Day 1 of arthritis. Thus, the increase in paw swelling from Day 1 in sFlt-PEG-treated mice was markedly reduced over the

(A)

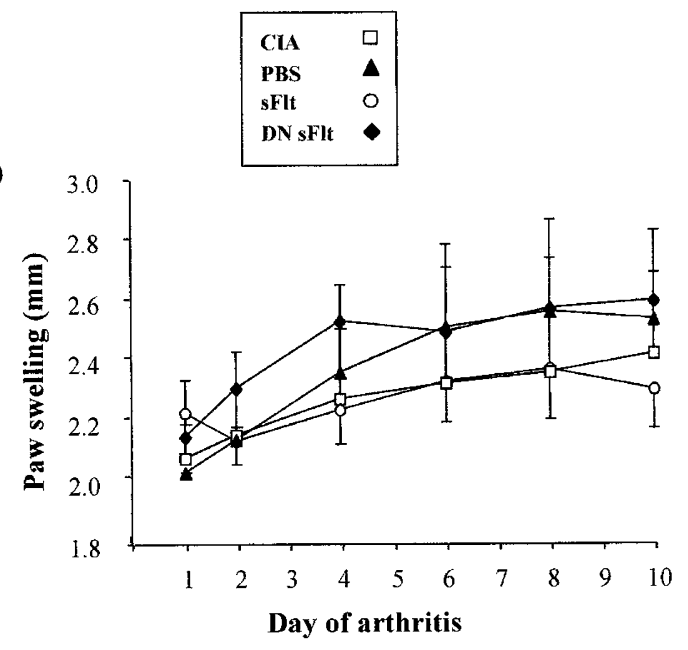

(B)

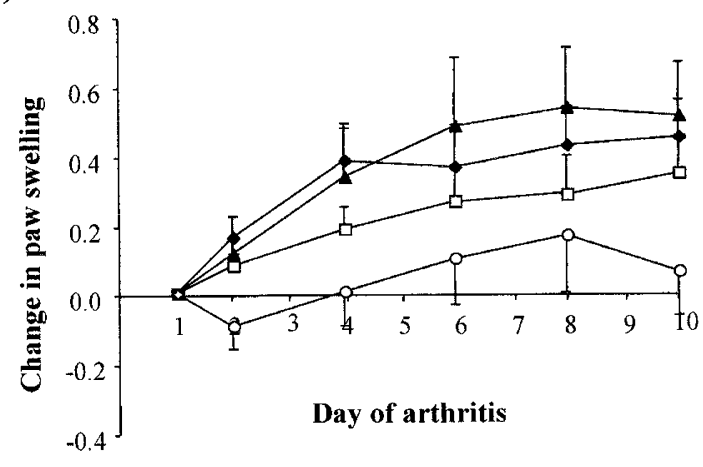

Figure 5.

Soluble VEGF receptor reduces the paw swelling in arthritic mice. Arthritis was induced in mice by immunization with bovine collagen. From the day of arthritis onset, mice were either untreated $(\square, n=16)$ or received the following treatments: sFlt-PEG $(\bigcirc, n=15)$ or denatured (DN) sFlt-PEG ( $\bullet$, $n=15)$, both at a dose of $300 \mu \mathrm{g}$, or vehicle $(\boldsymbol{\Delta}, \mathrm{PBS}, n=8)$, ip daily until Day 5 of arthritis. A, The paw swelling was measured daily until Day 10 or, B, expressed as change from Day 1. Results are mean \pm SEM.
10 days of arthritis compared with controls. For example, on Day 10 of arthritis, the increase in paw swelling from Day 1 in sFlt-PEG-treated mice was $0.1 \pm 0.1 \mathrm{~mm}$ (mean $\pm \mathrm{SEM}$ ), compared with $0.5 \pm 0.1$ for both PBS-treated and denatured sFlt-PEG-treated mice ( $p<0.05$ vs sFlt-PEG-treated mice) (Fig. 5B).

\section{Soluble VEGF Receptor sFIt-PEG Decreases Synovial Inflammation and Bone Destruction in CIA}

At the end of the experiment, paws were processed and assessed histologically for changes in the degree of inflammation and severity of cartilage and bone erosion. The histological changes after 10 days of arthritis were as follows: inflammation occurred in the synovial, subsynovial, and periosteal tissues and was characterized by edema and an inflammatory cell infiltrate containing a predominance of neutrophils (Fig. 6B), changes which were not identified in joints from normal mice (Fig. 6A); synovial membranes were generally degenerate and necrotic with areas of hyperplasia; development of pannus over the lateral borders of the articular cartilage resulted in erosion of the surface, and periosteal inflammation led to the erosion of bone adjacent to the articular surface. There was a very close relationship between the severity of inflammation and the degree of bone and cartilage erosion. Although there was evidence of bone erosion, none of the untreated animals exhibited bone destruction that could be described as "severe" at Day 10 of arthritis.

The effects of treatment with either heat-denatured sFlt-PEG or sFlt-PEG treatment on synovial inflammation and bone erosion were thus assessed (Fig. 6, C and $D$, respectively). The sections from treated animals (killed on Day 10 of arthritis) were graded in a blinded fashion, and the percentages of animals exhibiting normal, mild, minimal, moderate, or severe changes in bone/cartilage and synovitis were calculated (Fig. 7). In the sFlt-PEG-treated group, there was an increased number of animals in which the cartilage and bone architecture at joint interfaces appeared normal (Fig. 6D). For example, 55\% of examined paw sections from sFlt-PEG-treated mice exhibited no destruction of bone/cartilage, compared with only $25 \%$ of paws from untreated arthritic animals ( $p<$ 0.001 by $\chi^{2}$ test for trend) (Fig. 7A) and $25 \%$ of paws from animals treated with denatured sFlt-PEG ( $p<$ 0.01 by $\chi^{2}$ test for trend) (Fig. 7A). Similarly, there was a greater percentage of joints from the sFlt-PEGtreated group exhibiting no inflammatory infiltrates: $38 \%$ from sFlt-PEG-treated animals compared with $21 \%$ from untreated arthritic mice $\left(p<0.05\right.$ by $\chi^{2}$ test for trend) (Fig. 7B).

\section{Discussion}

VEGF, an important factor for endothelial cell proliferation and a key regulator of vasculogenesis in the embryo and angiogenesis in adult tissues (Brown et al, 1997), may potentially play a key role in both the joint swelling and synovial hyperproliferation that charac- 


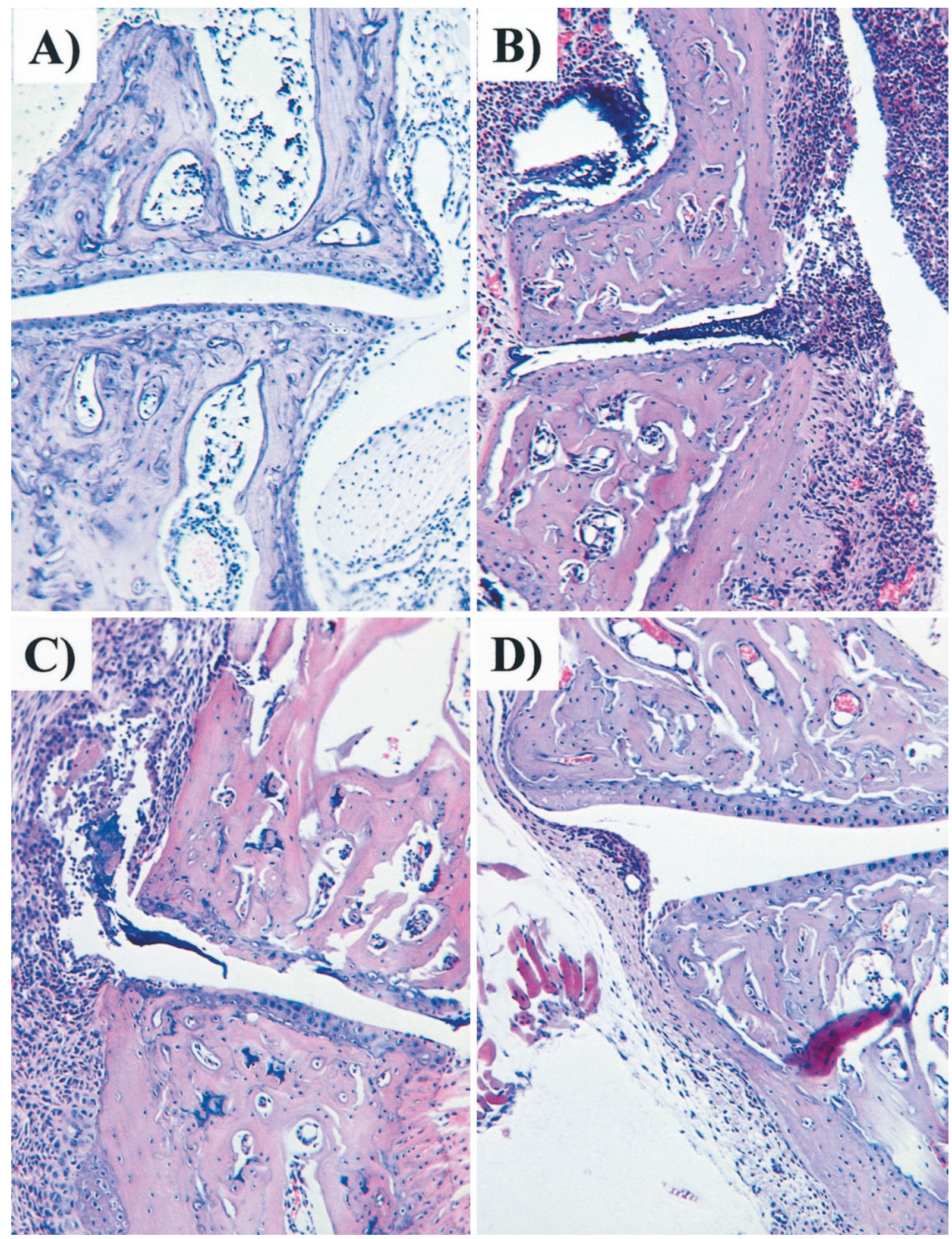

\section{Figure 6.}

Histological appearance of joint interfaces with or without arthritic disease. Mice were immunized with bovine collagen in complete Freund's adjuvant. On day of arthritis onset, mice were either untreated in the control group or treated with denatured sFlt-PEG or sFlt-PEG, both at a dose of $300 \mu \mathrm{g}$ ip, daily until Day 5 of arthritis. Paws were then fixed postmortem, processed, cut, and stained with hematoxylin and eosin for histological assessment. A, Normal joint from naive mouse. B, Severe arthritis at Day 10 after disease onset, demonstrating synovitis, cartilage loss, and bone erosion. C, Treatment with denatured sFlt-PEG did not inhibit inflammatory cell infiltration or cartilage/bone destruction. D, sFlt-PEG treatment resulted in development of only mild arthritis, ie, little synovitis and normal cartilage/bone. (Magnification $\times 115)$. 

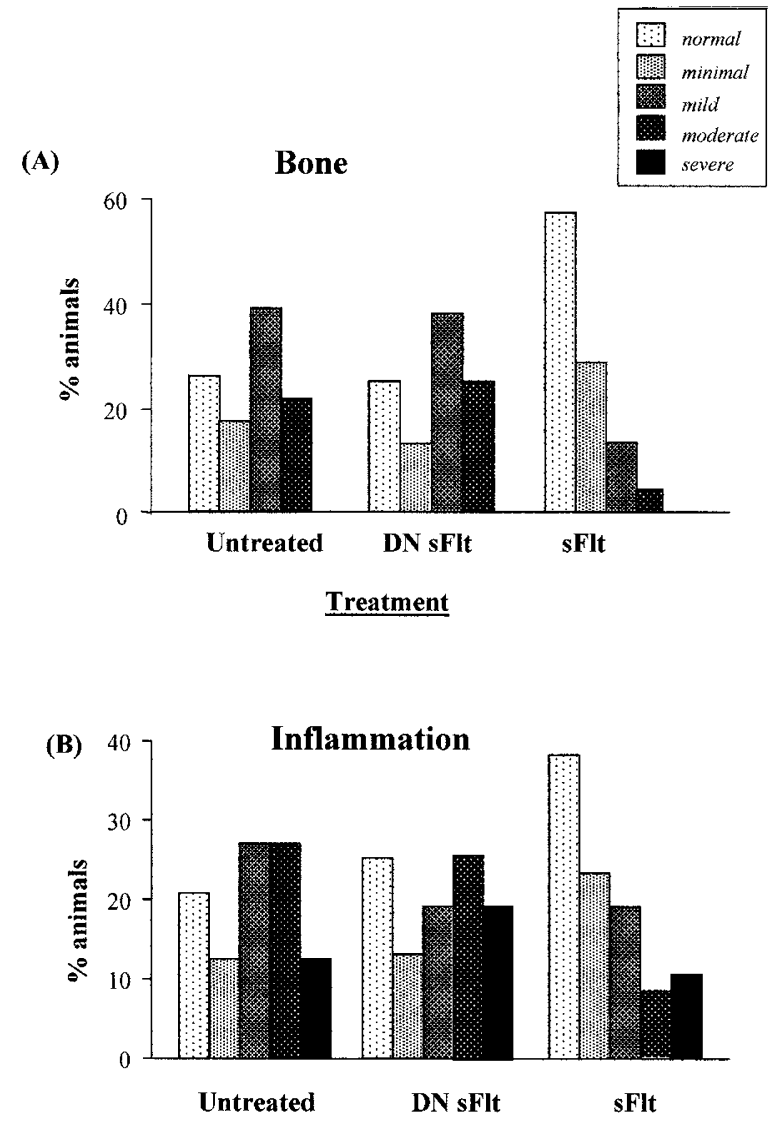

Treatment

\section{Figure 7.}

Administration of soluble VEGF receptor reduces bone/cartilage degradation and synovial inflammation. Mice were immunized with bovine collagen in complete Freund's adjuvant. On day of arthritis onset, mice were either untreated in the control group $(n=48)$ or treated with either denatured (DN) sFlt-PEG $(n=16)$ or sFlt-PEG $(n=47)$, both at a dose of $300 \mu \mathrm{g}$ ip, daily until Day 5 of arthritis. Paws were processed for histology, and histology sections were graded for the degree of $(A)$ bone/cartilage erosion and $(B)$ inflammation. Data are percentage of animals exhibiting normal, mild, minimal, moderate, and severe inflammation and bone/cartilage degradation.

terize RA. We have previously reported that cells isolated from synovial membrane samples, obtained from RA patients undergoing joint replacement, secrete VEGF in vitro (Paleolog et al, 1998). In addition, mRNA for both VEGF receptor subtypes Flt-1 and KDR on microvascular endothelial cells in RA synovium has been detected (Fava et al, 1994). However, studies involving RA patients are limited by the availability of samples, such as synovial tissue, and the effects of disease duration or therapeutic intervention. To this end, we have used the mouse CIA model, which resembles $R A$ in that it is an immune-driven disease with severe synovitis, pannus formation, destruction of the cartilage, and bone erosion, and it responds to TNF $\alpha$ blockade (Williams et al, 1992). We have investigated the expression of VEGF in the development of CIA and have examined the expression of VEGF by cells isolated from knee joints, both before and after onset of clinical symptoms of arthritis, in relation to the extent of disease.
The amount of VEGF produced increased as arthritis progressed, and it was observed that only cells from the joints of arthritic mice secreted significant amounts of VEGF protein in culture, with maximal release by Day 10 of disease. The extent of VEGF produced by cells from the knee joints of arthritic mice was dependent on the severity of disease, and the levels of VEGF correlated with both clinical scores and paw swelling. There was little or no detectable VEGF produced by cultures of synovial cells isolated from age-matched naive or sham-immunized animals or from mice immunized with collagen but killed prior to the onset of the disease. Thus in the mouse CIA model, production of detectable levels of VEGF protein was associated with onset of clinical symptoms of arthritis. This is similar to the human disease, in which VEGF expression is correlated with disease severity in patients with chronic RA (Paleolog et al, 1998). Although in patients with RA there are detectable levels of VEGF in the serum, we were unable to measure VEGF in the serum of mice with CIA (not shown). This is in contrast to reports describing measurable levels of serum VEGF in rat CIA (Oliver et al, 1994, 1998).

There is evidence in the literature that treatment of arthritis in animal models using compounds which exert nonspecific antiangiogenic, as well as other, effects significantly modulate disease with attenuation of neovascularization; eg, AGM-1470 and Taxol both attenuate experimental arthritis (Oliver et al, 1994; Peacock et al, 1992). The effect of selective blockade of the angiogenic factor, such as VEGF on progression of arthritic disease in an animal model, has not been described. It has been described, however, that antagonism of the vascular integrin $\alpha v \beta 3$ by intraarticular injections led to reduced vascularity in the synovium of rabbits after induction of arthritis with a combination of ovalbumin and FGF-2, and was associated with a significant decrease in all arthritic parameters, including joint swelling, synovitis, and synovial hypertrophy (Storgard et al, 1999). The study thus provided evidence that targeting new vessels may have a disease-modifying effect in arthritis. Hence, blockade of angiogenic pathways in RA is likely to be of therapeutic benefit.

We have now extended these observations by neutralizing the cytokine VEGF, using the soluble form of the Flt-1 VEGF receptor. VEGF binds to Flt-1 with a greater affinity than to KDR (Ortega et al, 1999; Shibuya, 1995, 1999). Soluble Flt is an alternatively spliced form of the Flt-1 receptor, containing six of the seven extracellular immunoglobulin-like domains, but lacking the transmembrane sequence and tyrosine kinase-containing region (Goldman et al, 1998; Kendall et al, 1996). The mechanism by which sFlt-PEG inhibits the action of VEGF probably involves not only sequestration of VEGF itself, but possibly also dimerization with full-length Flt-1 and KDR in a dominantnegative fashion (Kendall et al, 1996). The binding affinity of sFIt-PEG for VEGF is comparable to that of the full-length receptor, making this molecule an excellent candidate for blocking the activity of VEGF in vivo (Kendall et al, 1996). 
We report here for the first time that inhibition of VEGF using the soluble form of the human Flt-1 receptor significantly reduced disease severity in the mouse CIA model. In control groups, the severity of arthritis in all animals was maintained or increased over 10 days of disease. In contrast, in the group receiving anti-VEGF therapy, the effect of sFlt-PEG on CIA was observed even 5 days after cessation of treatment, with disease severity reduced in $33 \%$ of animals at Day 10 of arthritis. In contrast, heatdenatured sFIt-PEG was without effect on disease progression in vivo. Treatment with sFIt-PEG also significantly reduced the paw swelling. The paw thickness of the first affected paw was measured daily, and in $54 \%$ of animals who received sFlt-PEG, the swelling was decreased at Day 10 compared with Day 1. Furthermore, sFIt-PEG treatment significantly reduced the inflammatory cell infiltrate and synovitis in the joints as assessed by histological scoring. In addition to the mild inflammation or absence of inflammation in the paws, the integrity of the cartilage was preserved and there was little evidence of bone degradation. Thus, VEGF neutralization in vivo appears to modulate cellular influx in joints and protects against the destruction of joint architecture that is commonly seen in arthritis.

The principal role of VEGF in the pathogenesis of arthritis is likely to be increased blood vessel formation through up-regulated endothelial cell proliferation and reduced endothelial apoptosis (Benjamin and Keshet, 1997; Brock et al, 1991; Brown et al, 1997). Administration of sFlt-PEG reduced the clinical score and paw swelling, as well as the extent of synovitis and joint destruction, which is indicative of a suppression of the formation of the pannus. Blood vessels are required to nourish and maintain the pannus, and reduced synovial mass is almost certainly associated with a decrease in the total number of blood vessels, which was apparent in the histological sections examined. Unfortunately, it was not possible to quantify the effect of sFlt-PEG treatment on the vasculature specifically in the histology sections in the present study. Additionally, VEGF induces vascular permeability changes in the arthritic synovium, which lead to edema formation. However, the presence of other vasoactive mediators, such as histamine, plateletactivating factor, or bradykinin, may explain why the inhibitory effects of sFlt-PEG on paw swelling and clinical score were only partial, albeit significant. Moreover, the effects of VEGF may not be restricted to endothelial cell proliferation, but may extend to myeloid cells. For example, VEGF has been suggested to play a role in osteoclast recruitment (Niida et al, 1999). VEGF may also mediate chemotaxis of monocytes, and possibly neutrophils, through its action on Flt-1 receptor (Barleon et al, 1996). Thus, blockade of VEGF, in addition to down-regulating angiogenesis and vascular permeability, may also reduce macrophage recruitment and osteoclastic bone resorption, an area presently being addressed.

It is noteworthy that many of the antirheumatic drugs currently in clinical use or in trials apparently exert effects on the vasculature. For example, methotrexate, at doses which are achieved in the serum of RA patients treated with this drug, has been found to inhibit endothelial proliferation in vitro (Hirata et al, 1989). In a recent study, the disease-modifying antirheumatic drug bucillamine was found to inhibit VEGF production by RA synovial cells, although methotrexate was without effect (Nagashima et al, 1999). In addition, we have previously shown that treatment of human RA with monoclonal antibody to TNF $\alpha$ significantly reduced serum VEGF in a time- and dosedependent manner and correlated with alterations in disease parameters, such as C-reactive protein (CRP) levels and swollen joint counts (Paleolog et al, 1998). The reduction in circulating VEGF levels may lead to decreased joint vascularity and swelling. It is possible that a combination of anti-TNF $\alpha$ antibody and antiVEGF therapy, such as sFlt, may prove to be of greatest therapeutic benefit. Regional delivery of sFlt, for example, using adenovirus such as that reported to inhibit tumor growth (Kong et al, 1998), might also overcome systemic antiangiogenic effects. This is particularly important in view of the recent report that anti-VEGF treatment inhibited endochondral ossification and, consequently, bone lengthening (Gerber et al, 1999). However, the effects of anti-VEGF treatment on bone growth and cartilage resorption were reversible, suggesting that a study of the effects of VEGF blockade in arthritis warrants consideration.

In summary, although numerous angiogenic cytokines have been detected in RA tissue, the identification of VEGF in RA synovial membrane sections suggests that this cytokine may play a unique role in mediating angiogenesis in RA. We have shown in the present study that the amount of VEGF produced from synovial cells isolated from mouse knees is dependent on the severity of disease in the mouse model of CIA. This observation substantiates the hypothesis that VEGF production and arthritis manifestation are closely associated. Expression of VEGF is not merely a secondary result of disease progression because specific blockade of VEGF activity attenuated disease severity. Our results suggest that VEGF in particular, and possibly other components of the angiogenic pathway, are a potential therapeutic target in RA and that modulation of VEGF expression may synergize with anti-TNF $\alpha$ therapy, leading to long-term benefit.

\section{Materials and Methods}

\section{Induction and Assessment of Arthritis}

Arthritis was induced in male DBA/1 mice (8-10 weeks old) by intradermal injection of bovine type II collagen, purified in-house from articular cartilage as previously described (Miller, 1972), in complete Freund's adjuvant (Difco, Detroit, Michigan). Onset of arthritis was variable, occurring from Day 14 up to Day 40 after immunization with collagen. Arthritis onset was considered to occur on the day that swelling and/or erythema were observed. Clinical score, which is a composite of disease severity and the number of limbs 
affected, was monitored daily from onset of disease and used as an assessment of disease progress. The scoring used was as follows: 0 , normal; 1 , slight swelling with erythema; 2, pronounced swelling; 3, joint rigidity. In addition, the extent of paw swelling was followed by measuring the paw thickness with callipers. The paw swelling reflects the degree of edema in affected limbs. Arthritis increased progressively over 10 days as reflected by both clinical score and paw swelling, which mirrored one another.

\section{Synovial Membrane Cell Preparation and Culture}

The hind limbs were removed and the synovium was dissected from the knee joints with the aid of a dissecting microscope, according to methods described previously (Butler et al, 1997; Malfait et al, 1998). The synovial tissue was digested with collagenase $A(1 \mathrm{mg} / \mathrm{ml})$ (Boehringer Mannheim, Lewes, United Kingdom) and DNAase (type IV $150 \mu \mathrm{g} / \mathrm{ml}$ ) (Sigma, Aldrich, United Kingdom) in the presence of polymixin B sulfate (Sigma) to produce a single cell suspension. The synovial cells were then washed and resuspended in RPMI (PAA Laboratories, Linz, Austria) containing $10 \%$ fetal calf serum (Sigma). Cells were cultured at a density of $4 \times 10^{6}$ cells in $30 \mathrm{~mm}^{2}$ wells in flat-bottomed plates at $37^{\circ} \mathrm{C}$. After 24 hours, the supernatants were removed and stored at $-20^{\circ} \mathrm{C}$ until further assay. VEGF in synovial membrane cell culture supernatants was assayed by ELISA for mouse VEGF (R\&D Systems, Oxford, United Kingdom) (sensitivity, $5 \mathrm{pg} / \mathrm{ml})$.

\section{VEGF mRNA Isolation and PCR Amplification}

Synovial cells were isolated from the knee joints of naive mice, those without arthritis (immunized with collagen 14 days previously), and from mice with arthritis 5 days after disease onset. Cells were frozen at $-70^{\circ} \mathrm{C}$ until further use. Total cytoplasmic RNA was prepared from the frozen cells using the RNeasy protocol (Qiagen, Hilden, Germany). First strand cDNA was synthesised from $0.6 \mu \mathrm{g}$ total RNA using the SUPERSCRIPT pre-amplification system (Gibco, Paisley, United Kingdom) followed by the PCR amplification reaction. The PCR was subjected to 30 temperature cycles as follows: $92^{\circ} \mathrm{C}$ for 2 minutes to denature, annealing at $55^{\circ} \mathrm{C}$ for 30 seconds, and extension at $72^{\circ} \mathrm{C}$ for 30 seconds. The final extension step was carried out for 5 minutes. The following synthetic oligonucleotide murine VEGF primers for amplification were used: ATGAACTTTCTGCTCTCTTGG (forward) and TCACCGCCTTGGCTTGTCACA (reverse) (Gibco). The products were separated on $2 \%$ agarose gels and visualized by ethidium bromide staining.

\section{Cloning, Expression, and Purification of Soluble VEGF Receptor Flt-1}

A soluble form of the VEGF receptor Flt-1, corresponding to amino acids 1-656 of the full-length receptor, equivalent to a 27 amino acid signal sequence and the N-terminal immunoglobulin-like do- mains 1 to 6 , plus a unique 31 amino acid C-terminal region, was expressed in baculovirus-infected High5 cells. A total of 5 litres of cell culture supernatant was loaded onto a 10-ml heparin Sepharose column, preequilibrated in $10 \mathrm{~mm}$ sodium phosphate, $\mathrm{pH}$ 6.2. The column was washed with $60 \mathrm{ml}$ of $10 \mathrm{~mm}$ sodium phosphate, $\mathrm{pH} 6.2$, containing $0.6 \mathrm{M} \mathrm{NaCl}$. Soluble Flt-1 receptor was eluted with $10 \mathrm{~mm}$ sodium phosphate, $\mathrm{pH} 6.2$, containing $1 \mathrm{M} \mathrm{NaCl}$ and stored at $-80^{\circ} \mathrm{C}$. Yields ranged from 2 to $10 \mathrm{mg} / \mathrm{l}$ of cell culture medium. The protein concentration was determined by amino acid composition analysis to give a product corresponding to amino acids 27-687 of the published peptide sequence of soluble human vascular endothelial cell growth factor receptor Flt-1 [Accession number AAC50060 (Kendall and Thomas, 1993)].

To PEG-link the purified soluble Flt-1, $20 \mathrm{ml}$ batches were thawed and diluted 1:1 with $0.8 \mathrm{M}$ sodium borate, $\mathrm{pH}$ 8.95. Methoxypolyethylene glycol p-nitrophenyl carbonate (Sigma) at 10-fold molar excess of primary amine groups on the soluble Flt-1 was added to $5 \mathrm{ml}$ of water. The PEGylation reaction was allowed to occur for 2 hours before termination of the reaction by the addition of $5 \mathrm{ml} 0.1 \mathrm{M}$ ethanolamine, $\mathrm{pH}$ 8.0. The PEG-linked soluble Flt-1 was concentrated to approximately $1 \mathrm{mg} / \mathrm{ml}$ in an Amicon-stirred cell and dialysed extensively against $50 \mathrm{~mm}$ sodium phosphate, $\mathrm{pH} 7.2$, containing $0.15 \mathrm{M} \mathrm{NaCl}$, to remove $\mathrm{p}$-nitrophenol. The concentration of the final product was determined by amino acid composition analysis, and storage was at $-80^{\circ} \mathrm{C}$.

To examine the activity of sFlt-PEG, the proliferation of HUVEC in response to growth factors was assessed. Addition of sFlt-PEG markedly inhibited proliferation of HUVEC in response to VEGF, with an $\mathrm{IC}_{50}$ value of $\leq 4 \mathrm{ng} / \mathrm{ml}$ (not shown). In contrast, proliferation of HUVEC induced by epidermal growth factor (EGF) and fibroblast growth factor (FGF)-2 was unaffected $\left(\mathrm{IC}_{50}\right.$ vs both EGF and FGF-2 $\left.>100 \mathrm{ng} / \mathrm{ml}\right)$. For comparison, a sample of sFlt-PEG was heated in a boiling water bath for 10 minutes. Heat-inactivation of sFIt-PEG abolished the inhibitory effect in terms of VEGF-stimulated proliferation of the HUVEC $\left(\mathrm{IC}_{50}\right.$ of heat-inactivated sFlt-PEG $>100 \mathrm{ng} / \mathrm{ml}$ ).

\section{Treatment of CIA with Soluble VEGF Receptor sFIt-PEG}

Blockade of VEGF activity in vivo was achieved by daily administration of sFlt-PEG, which was administered intraperitoneally at a dose of $100 \mu \mathrm{g}$ or $300 \mu \mathrm{g}$ per animal, equivalent to 5 or $15 \mathrm{mg} / \mathrm{kg}$. In one experiment, animals were dosed on the day of arthritis onset and every other day until Day 10 with $100 \mu \mathrm{g}$ sFIt-PEG. In a separate experiment, treatment at 300 $\mu \mathrm{g}$ was initiated on Day 1 of arthritis onset and subsequently every day until Day 5 . Animals were then left untreated until Day 10 when the experiment was terminated. Throughout disease duration the animals were scored for clinical symptoms of arthritis, and a measure of their paw swelling was taken as described earlier. Control animals received heat-denatured sFlt$P E G$ with the same dosing regimen. Administration of 
the denatured protein had no adverse affects, and arthritis development was unaltered in the mice.

\section{Histopathology}

Hind paws were removed at the end of the experiment and fixed in $10 \%$ buffered formalin. Tissue was decalcified in 5\% (w/v) EDTA and processed prior to embedding in paraffin wax. Sections were cut and stained with hematoxylin and eosin according to standard procedure. The joints of the foot and ankle were then examined microscopically, and subjectively graded for the degree of inflammation (particularly synovitis with pannus formation), and for bone and cartilage erosion, as follows: normal, no change; minimal, a few small foci/areas of synovitis/cartilage loss; mild, some synovitis/bone erosions, with up to $20 \%$ involvement or subjective equivalent; moderate, synovitis/bone erosions, with up to $50 \%$ involvement or subjective equivalent; severe, synovitis, extensive erosions and disrupted joint architecture, in more than $50 \%$ involvement or subjective equivalent. Analyses were performed in a blinded fashion by an observer unaware of the clinical status of the animal or the treatment received.

\section{Statistical Analysis}

Statistical analyses were performed using the GraphPad Prism software package (GraphPad Software, San Diego, California). Student's $t$ test and MannWhitney $U$ test were used to compare the differences between various groups of animals for Gaussian and non-Gaussian populations, respectively. Welch's modification of the $t$ test was used when the variance of the two populations was unequal. Two-way ANOVA was used to determine the effect of treatment over 10 days. The $\chi^{2}$ test for trend was used to compare histological changes after anti-VEGF treatment. $P$ values of less than 0.05 were considered to be statistically significant. The non-parametric Spearman correlation was used to examine the degree of linear association between VEGF production and paw thickness or clinical scores. It is based on ranking the variables and makes no assumption about the distribution.

\section{Acknowledgements}

This research was supported by the Arthritis Research Campaign of Great Britain and AstraZeneca Pharmaceuticals, Alderly Park, Macclesfield, United Kingdom. The authors would like to thank the following: at the Kennedy Institute of Rheumatology, Ms. Sylvia Young for expert technical assistance and Mr. Philip Connolly for help with the histological studies; at AstraZeneca Pharmaceuticals, Mr. Rick Davies and Ms. Judith Stanway for synthesis and PEGylation of sFlt, and Dr. Russell Westwood for the histopathological assessments. Finally, the authors are indebted to Professor R. N. Maini for his interest and support.

\section{References}

Barleon B, Sozzani S, Zhou D, Weich HA, Mantovani A, and Marme D (1996). Migration of human monocytes in response to vascular endothelial growth factor (VEGF) is mediated via the VEGF receptor flt-1. Blood 87:3336-3343.

Benjamin LE and Keshet E (1997). Conditional switching of vascular endothelial growth factor (VEGF) expression in tumors: Induction of endothelial cell shedding and regression of hemangioblastoma-like vessels by VEGF withdrawal. Proc Natl Acad Sci U S A 94:8761-8766.

Brock TA, Dvorak HF, and Senger DR (1991). Tumorsecreted vascular permeability factor increases cytosolic $\mathrm{Ca}^{2+}$ and von Willebrand factor release in human endothelial cells. Am J Pathol 138:213-221.

Brown LF, Detmar M, Claffey K, Nagy JA, Feng D, Dvorak AM, and Dvorak HF (1997). Vascular permeability factor/ vascular endothelial growth factor: A multifunctional angiogenic cytokine. EXS 79:233-269.

Butler DM, Malfait AM, Mason LJ, Warden PJ, Kollias G, Maini RN, Feldmann M, and Brennan FM (1997). DBA/1 mice expressing the human TNF-alpha transgene develop a severe, erosive arthritis: Characterization of the cytokine cascade and cellular composition. J Immunol 159:2867-2876.

Claffey KP, Wilkison WO, and Spiegelman BM (1992). Vascular endothelial growth factor. Regulation by cell differentiation and activated second messenger pathways. J Biol Chem 267:16317-16322.

Fava RA, Olsen NJ, Spencer-Green G, Yeo KT, Yeo TK, Berse B, Jackman RW, Senger DR, Dvorak HF, and Brown LF (1994). Vascular permeability factor/endothelial growth factor (VPF/VEGF): Accumulation and expression in human synovial fluids and rheumatoid synovial tissue. J Exp Med 180:341-346.

Feldmann M, Brennan FM, and Maini RN (1996). Rheumatoid arthritis. Cell 85:307-310.

Gerber HP, Vu TH, Ryan AM, Kowalski J, Werb Z, and Ferrara N (1999). VEGF couples hypertrophic cartilage remodeling, ossification, and angiogenesis during endochondral bone formation. Nat Med 5:623-628.

Goldman CK, Kendall RL, Cabrera G, Soroceanu L, Heike Y, Gillespie GY, Siegal GP, Mao X, Bett AJ, Huckle WR, Thomas KA, and Curiel DT (1998). Paracrine expression of a native soluble vascular endothelial growth factor receptor inhibits tumor growth, metastasis, and mortality rate. Proc Natl Acad Sci U S A 95:8795-8800.

Hirata S, Matsubara T, Saura R, Tateishi H, and Hirohata K (1989). Inhibition of in vitro vascular endothelial cell proliferation and in vivo neovascularization by low-dose methotrexate. Arthritis Rheum 32:1065-1073.

Kendall RL and Thomas KA (1993). Inhibition of vascular endothelial cell growth factor activity by an endogenously encoded soluble receptor. Proc Natl Acad Sci U S A 90: 10705-10709.

Kendall RL, Wang G, and Thomas KA (1996). Identification of a natural soluble form of the vascular endothelial growth factor receptor, FLT-1, and its heterodimerization with KDR. Biochem Biophys Res Commun 226:324-328.

Koch AE (1998). Review: Angiogenesis: Implications for rheumatoid arthritis. Arthritis Rheum 41:951-962. 
Koch AE, Harlow LA, Haines GK, Amento EP, Unemori EN, Wong WL, Pope RM, and Ferrara N (1994). Vascular endothelial growth factor. A cytokine modulating endothelial function in rheumatoid arthritis. J Immunol 152:4149-4156.

Kong HL, Hecht D, Song W, Kovesdi I, Hackett NR, Yayon A, and Crystal RG (1998). Regional suppression of tumor growth by in vivo transfer of a cDNA encoding a secreted form of the extracellular domain of the flt-1 vascular endothelial growth factor receptor. Hum Gene Ther 9:823-833.

Malfait AM, Butler DM, Presky DH, Maini RN, Brennan FM, and Feldmann M (1998). Blockade of IL-12 during the induction of collagen-induced arthritis (CIA) markedly attenuates the severity of the arthritis. Clin Exp Immunol 111:377-383.

Miller EJ (1972). Structural studies on cartilage collagen employing limited cleavage and solubilization with pepsin. Biochemistry 11:4903-4909.

Nagashima M, Yoshino S, Aono H, Takai M, and Sasano M (1999). Inhibitory effects of anti-rheumatic drugs on vascular endothelial growth factor in cultured rheumatoid synovial cells. Clin Exp Immunol 116:360-365.

Nagashima M, Yoshino S, Ishiwata T, and Asano G (1995). Role of vascular endothelial growth factor in angiogenesis of rheumatoid arthritis. J Rheumatol 22:1624-1630.

Neufeld G, Cohen T, Gengrinovitch S, and Poltorak Z (1999). Vascular endothelial growth factor (VEGF) and its receptors. FASEB J 13:9-22.

Niida S, Kaku M, Amano H, Yoshida H, Kataoka H, Nishikawa S, Tanne K, Maeda N, Nishikawa SI, and Kodama H (1999). Vascular endothelial growth factor can substitute for macrophage colony-stimulating factor in the support of osteoclastic bone resorption. J Exp Med 190:293-298.

Oliver SJ, Banquerigo ML, and Brahn E (1994). Suppression of collagen-induced arthritis using an angiogenesis inhibitor, AGM-1470, and a microtubule stabilizer, taxol. Cell Immunol 157:291-299.

Oliver SJ, Cheng TP, Banquerigo ML, and Brahn E (1998). The effect of thalidomide and 2 analogs on collagen induced arthritis. J Rheumatol 25:964-969.

Ortega N, Hutchings $\mathrm{H}$, and Plouet $\mathrm{J}$ (1999). Signal relays in the VEGF system. Front Biosci 4:D141-D152.

Paleolog EM (1996). Angiogenesis: A critical process in the pathogenesis of RA-a role for VEGF? $\mathrm{Br} J$ Rheumatol 35:917-919.

Paleolog EM and Fava RA (1998). Angiogenesis in rheumatoid arthritis: Implications for future therapeutic strategies. Springer Semin Immunopathol 20:73-94.
Paleolog EM and Miotla JM (1998). Angiogenesis in arthritis: Role in disease pathogenesis and as a potential therapeutic target. Angiogenesis 2:295-307.

Paleolog EM, Young S, Stark AC, McCloskey RV, Feldmann $M$, and Maini RN (1998). Modulation of angiogenic vascular endothelial growth factor by tumor necrosis factor alpha and interleukin-1 in rheumatoid arthritis. Arthritis Rheum 41: 1258-1265.

Peacock DJ, Banquerigo ML, and Brahn E (1992). Angiogenesis inhibition suppresses collagen arthritis. J Exp Med 175:1135-1138.

Salmi M, Rajala P, and Jalkanen S (1997). Homing of mucosal leukocytes to joints. Distinct endothelial ligands in synovium mediate leukocyte-subtype specific adhesion. J Clin Invest 99:2165-2172.

Shibuya M (1995). Role of VEGF-flt receptor system in normal and tumor angiogenesis. Adv Cancer Res 67:281-316.

Shibuya M, Ito N, and Claesson-Welsh L (1999). Structure and function of vascular endothelial growth factor receptor-1 and -2. Curr Top Microbiol Immunol 237:59-83.

Shima DT, Kuroki M, Deutsch U, Ng YS, Adamis AP, and D'Amore PA (1996). The mouse gene for vascular endothelial growth factor. Genomic structure, definition of the transcriptional unit, and characterization of transcriptional and posttranscriptional regulatory sequences. J Biol Chem 271:38773883.

Storgard CM, Stupack DG, Jonczyk A, Goodman SL, Fox RI, and Cheresh DA (1999). Decreased angiogenesis and arthritic disease in rabbits treated with an alphavbeta3 antagonist. J Clin Invest 103:47-54.

Taylor P, Patel S, Paleolog E, McCloskey RV, Feldmann M, and Maini RN (1998). Reduced synovial vascularity following TNF $\alpha$ blockade in rheumatoid arthritis (abstract 1577). Arthritis Rheum 41(Suppl 9):S295.

Vasir B, Aiello LP, Yoon KH, Quickel RR, Bonner-Weir S, and Weir GC (1998). Hypoxia induces vascular endothelial growth factor gene and protein expression in cultured rat islet cells. Diabetes 47:1894-1903.

Williams RO, Feldmann M, and Maini RN (1992). Anti-tumor necrosis factor ameliorates joint disease in murine collageninduced arthritis. Proc Natl Acad Sci U S A 89:9784-9788. 